\title{
8
}

\section{EL MODELO DE GUÍA DOCENTE GEA: UN RECURSO PARA ORIENTAR EL PROCESO DE APRENDIZAJE Y MEJORAR LA TUTORÍA ACADÉMICA DEL ALUMNADO}

\section{THE GEA TEACHING GUIDE MODEL:} A RESOURCE TO GUIDE THE LEARNING PROCESS AND IMPROVE THE ACADEMIC TUTORING OF STUDENTS

\author{
Pedro Álvarez-Pérez \\ palvarez@ull.edu.es \\ David López-Aguilar \\ dlopez@ull.edu.es \\ Rocío Peña Vázquez \\ ropeva@ull.edu.es
}

Universidad de La Laguna, España

https://doi.org/10.25145/b.innovaull.2019.008 


\section{RESUMEN}

Desde la implantación del Espacio Europeo de Educación Superior, uno de los recursos básicos de la enseñanza universitaria es la guía docente, en la que se estructuran los elementos básicos y se planifica el proceso de enseñanza-aprendizaje de cada asignatura. La guía docente constituye para el profesorado una herramienta importante para estructurar el programa que pondrá en práctica en cada asignatura. Pero también es un documento de referencia fundamental para el alumnado, puesto que en la guía docente se incorpora información clave acerca de los elementos básicos del proceso de enseñanza-aprendizaje de cada materia. Las valoraciones previas llevadas a cabo por el profesorado y alumnado de la ULL respecto al modelo de guía docente actual, ha sido el punto de partida para proponer, por parte del grupo de Innovación Docente GICA, el modelo de guía de enseñanza-aprendizaje (GEA). En este modelo, que se ajusta a la normativa de la enseñanza universitaria, se mantienen algunos apartados del modelo de guía docente actual, se eliminan otros no obligatorios y se incorporan nuevos apartados destinados a facilitar su integración en el proceso formativo, guiar al alumnado en su proceso de aprendizaje y contribuir a una mejor atención personalizada.

PALABRAS CLAVE: Guía docente; planificación de la enseñanza; orientación al alumnado; guía de enseñanza-aprendizaje.

\section{ABSTRACT}

Since the introduction of the European Higher Education Area, one of the basic resources of university education is the teaching guide, in which the basic elements are structured and the teaching-learning process of each subject is planned. The teaching guide is an important tool for teachers to structure the program that will be put into practice in each subject. But it is also a fundamental reference document for students, since the teaching guide incorporates key information about the basic elements of the teaching-learning process of each subject. The previous evaluations carried out by the teachers and students of the ULL regarding the current teaching guide model, has been the starting point to propose, on the part of the GICA Teaching Innovation group, the teaching-learning guide model (GEA) . In this model, which conforms to the regulations of university education, some sections of the current teaching guide model are maintained, other non-compulsory ones are eliminated and new sections are incorporated to facilitate their integration in the training process, guide to the students in their learning process and contribute to a better personalized attention.

KEYWORDS: Teaching guide; teaching planning; orientation to students; teachinglearning guide.

\section{INTRODUCCIÓN: LA GUÍA DE ENSEÑANZA-APRENDIZAJE (GEA)}

En los últimos años, con la implantación del Espacio Europeo de Educación Superior, se ha venido destacando la importancia que tienen las guías docente como herramienta básica para guiar el proceso de 
aprendizaje el alumnado (García, 2008; Marcelo, Yot, Mayor, Sánchez, Murillo, Rodríguez y Pardo, 2014). La elaboración de la guía docente supone un ejercicio de planificación y reflexión, para relacionar los objetivos del aprendizaje con las competencias que se pretenden desarrollar en la asignatura, a través de las actividades formativas y empleando las estrategias adecuadas de evaluación para el seguimiento y control del aprendizaje. Esta planificación se ocupa de aspectos como la apropiada distribución de la carga de trabajo del alumnado, la organización temporal de las actividades formativas, los contenidos y materiales docentes, los plazos de entrega de trabajos e informes, la metodología de evaluación y todo lo que el estudiante necesita conocer para superar una asignatura (Zabalza, 2003; Sánchez, Ruiz y Pascual, 2011). Se trata de un recurso importante y un documento de referencia necesario para el alumnado, que le permite optimizar su trabajo y obtener el máximo aprovechamiento del proceso de enseñanza-aprendizaje.

Dada la relevancia que consideramos tiene la guía docente en la enseñanza universitaria, en los últimos años el grupo de innovación GICA está llevando a cabo una línea de trabajo orientada a la mejora de este recurso y se ha propuesto el modelo GEA (Guía de Enseñanza Aprendizaje), con el que se pretende que la guía docente no sea solo un documento administrativo, sino una herramienta integrada en los procesos de enseñanza-aprendizaje. La propuesta de este modelo de guía docente, parte de la idea de entender esta herramienta didáctica como un documento que recoge la información básica, que permite al estudiante comprender las características principales del proceso de enseñanzaaprendizaje de una asignatura. La guía docente ha sufrido modificaciones a lo largo del tiempo, debido a las cambiantes normativas que en ella han influido y que cada vez más la han enfocado hacia una planificación muy detallada de cada asignatura que han hecho que, por un lado, el profesorado la pueda ver como un requisito administrativo y el estudiante como una mera herramienta de garantía de cumplimiento de lo expuesto en la misma (Yaniz, 2006).

La propuesta de modelo de guía docente que aquí se ofrece, tiene como eje central el proceso de enseñanza-aprendizaje, lo que implica que el profesorado realice un verdadero ejercicio de planificación y reflexión en su elaboración, relacionando los objetivos del aprendizaje, con las competencias que se pretende que adquiera el estudiante, las actividades formativas a desarrollar y la evaluación. Por otro lado, la guía docente debe suponer para el estudiante la referencia que le permita optimizar su trabajo, obteniendo el máximo aprovechamiento del proceso de enseñanza-aprendizaje de la asignatura. Esta sería la principal razón por la que a este modelo de guía docente se le ha denominado Guía de Enseñanza y Aprendizaje (GEA). 


\section{ESTRUCTURA Y APARTADOS DE LA GEA}

La estructura de la GEA se ha establecido atendiendo a las normativas nacionales, regionales y de la Universidad de La Laguna (ULL) implantadas para el diseño de las guías docentes. En este sentido, la GEA recoge, por un lado, todos los apartados de obligado cumplimiento derivados de las actuales normativas, como aquellas otras propuestas aportadas como recomendaciones. De esta manera, en la GEA se incluye, tanto los datos que describen la asignatura y el profesorado que la va impartir, como los aspectos más relevantes relacionados con el proceso de enseñanza-aprendizaje de la propia asignatura (competencias y resultados de aprendizaje, contenidos desarrollados, actividades formativas y la evaluación que indica si se han adquirido las competencias establecidas en la asignatura). La estructura GEA se divide en tres bloques de contenidos:

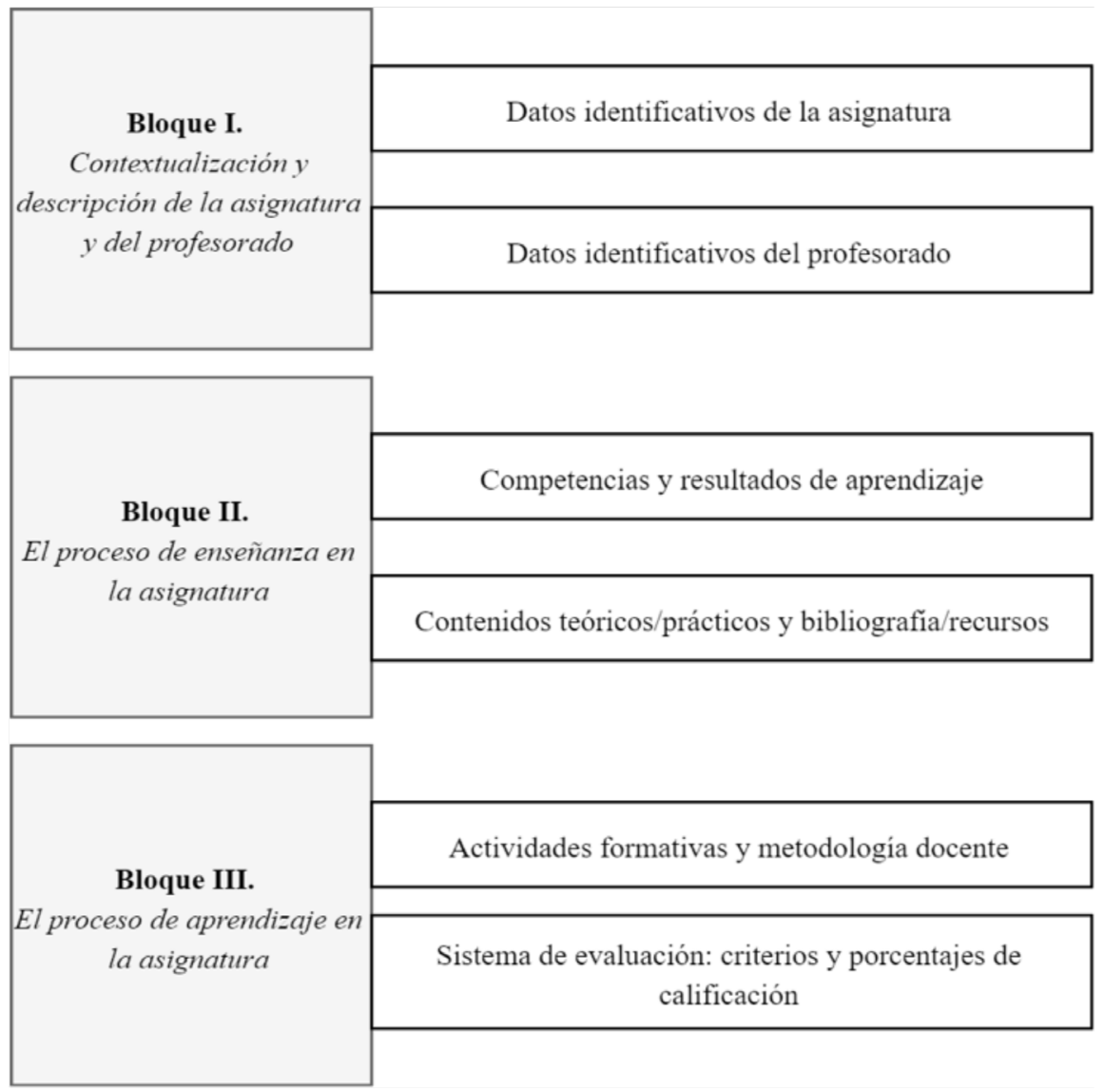

Figura 1.

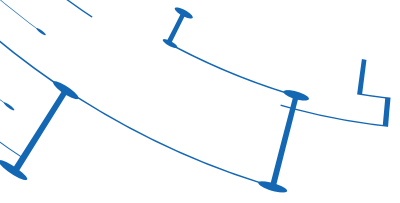


En cada uno de los bloques se desarrollan los siguientes contenidos:

\section{BLoque I: ConteXtuALIZACIÓN Y DESCRIPCIÓN DE LA ASIGNATURA Y DEL PROFESORADO}

\section{Datos identificativos de la asignatura.}

En este apartado se recoge la información relativa a la asignatura, identificándola (nombre, código, etc.), contextualizándola en la titulación e indicando las características de mayor relevancia para el estudiante (horario de impartición de la materia, aulas, etc.).

Este apartado se caracteriza porque la mayor parte del mismo será de contenido fijo derivado de las memorias de verificación/ modificación y la parte variable no dependerá de la elaboración del profesorado ya que ésta vendrá dada por el propio centro mediante la generación de hipervínculos.

\section{Datos identificativos del profesorado.}

- En este apartado se recoge la información relativa al profesorado que imparte la asignatura, contextualizándolo dentro del departamento al cual pertenece e indicando las características más importantes a conocer por el estudiante en el desarrollo de su docencia, así como su aportación en el desarrollo de la asignatura.

- Aunque este apartado debe ser elaborado por el profesorado que imparte la asignatura en un primer momento, una vez incluida la información, esta pasará a ser fija (excepto por motivo de cambio de profesorado). En concreto, la información que se integra en este apartado es la siguiente: nombre y apellidos del profesorado, departamento y área de conocimiento, correo electrónico, teléfono, horario y lugar de tutorías, currículum vitae relacionado con la asignatura.

\section{BLOQUE II: El PROCESO dE ENSEÑANZA EN LA ASIGNATURA}

- El objetivo principal de este bloque es indicar los aspectos principales que van a tener lugar en el desarrollo de la asignatura, qué conocimientos va a adquirir el estudiante con dicha enseñanza y cuáles son los recursos bibliográficos en los que se puede apoyar para ello.

- Este bloque es de contenido fijo, derivado de las memorias de verificación/modificación, excepto en aquellos casos en el que en dichas memorias no aparecen asignadas a las asignaturas algunos de los apartados. 


\section{Breve presentación de la asignatura.}

- Se trata de resumir en pocas líneas las ideas fundamentales que se quiere que el estudiante conozca de la asignatura: cuál es su principal objetivo, qué se aprenderá con esta asignatura, si aporta conocimientos necesarios para cursar otras asignaturas, su importancia para el ejercicio profesional, etc., de manera que ayude al estudiante a entender el papel de la asignatura en el proyecto formativo de la titulación.

\section{Competencias y RESUltAdos DE APRENDIZAJE DE LA ASIGNATURA.}

- En este apartado se deberán especificar todos los tipos de competencias (básicas, transversales y/o generales, y específicas) de la asignatura, así como los resultados concretos que se espera alcanzar de su aprendizaje. La finalidad de establecer las competencias y resultados de aprendizaje en este mismo apartado es la de que los estudiantes conozcan cuáles son los conocimientos, habilidades y destrezas (competencias) que adquirirán con la asignatura y cuáles son las actuaciones concretas que con estas esperamos que sean capaces de desarrollar, en qué contexto y a qué nivel (resultados de aprendizaje).

- El listado de todas las competencias (básicas, transversales y/o generales, y específicas) y de los resultados de aprendizaje de la titulación aparecen establecidas en su memoria de Verificación o Modificación. Se deberá escoger y nombrar todas las establecidas para la asignatura relacionando las competencias con los resultados de aprendizaje.

- Las competencias vinculadas a una asignatura deben transcribirse directamente de la ficha correspondiente de la memoria de verificación o modificación del título. No se pueden modificar, salvo que la modificación venga motivada por la introducción de cambios en el plan de estudios de la titulación.

\section{Contenidos teóricos/Prácticos y bibliografía/ReCURSOS.}

-Este apartado hace referencia a los contenidos de la asignatura y su agrupación por módulos y unidades didácticas o temas, aportando estructura a este apartado. Aunque los contenidos teóricos/prácticos en este apartado se rellenarían a partir de las memorias de verificación/modificación, hay que tener en cuenta que los mismos deben haberse establecidos atendiendo a las competencias y a los resultados de aprendizaje de la asignatura. 
- La selección de estos contenidos que constituirán el temario de la asignatura se elegirá teniendo en cuenta los descriptores de contenido de cada titulación, las competencias a alcanzar (propuestas en el plan de estudios) y los resultados de aprendizaje esperados. Los contenidos no son un fin en sí mismos, lo seleccionamos porque contribuye al desarrollo intelectual o preparación profesional del estudiante, lo que implica que aborda no sólo aspectos técnicos, sino también aspectos éticos y morales. Deben desarrollar habilidades intelectuales de un nivel superior como razonamiento, resolución de problemas, pensamiento crítico, creatividad, etc.

- El temario debe ser proporcional a los créditos de la asignatura, seleccionando contenidos relevantes de forma realista y permitiendo que el alumnado profundice en ellos. Se podría distinguir entre contenidos básicos o imprescindibles y aquellos complementarios. Se pueden utilizar acrónimos para facilitar su referencia en otros apartados y ayudar al estudiante en su identificación. Es importante indicar si hay contenidos prácticos (en aula, o en laboratorios o en sala de demostraciones, salas de informática, etc.), su nombre, una breve descripción se su contenido y si necesita ser evaluada.

- El tipo de práctica debe incorporarse en la guía docente: comentario de texto, análisis de caso, visionado y comentario de documental o película, etc. Es relevante especificar aquellos contenidos que van a ser realizados en otro idioma y además es útil para intercambios con otros países replicar todos los contenidos en inglés. Se debe seleccionar la bibliografía y los recursos más significativos para los estudiantes. Este apartado se puede organizar por secciones:

- Bibliografía general/básica de la asignatura: se usa para preparar la asignatura completa o partes importantes de la misma. Abarca libros, apuntes, escritos, artículos, etc. Es muy importante que sean accesibles para el estudiante. Si fuese muy difícil de conseguir, no debería formar parte de este grupo de bibliografía general. Se aconseja ordenarla según el temario para facilitar el seguimiento por parte del alumnado.

- Bibliografía específica: ordenada por temas, es la bibliografía que se emplearía si se quisiera ampliar y profundizar en cada tema. Al ser más específicas, servirán de apoyo para realización de trabajos o pueden ser similares a las generales, pero con enfoques diferentes.

- Recursos en red y otros recursos: páginas web recomendadas, material multimedia complementario, etc. Se aconseja incluir el aula virtual como recurso en red.

- Es fácil que dispongamos de mucha bibliografía que supera el número recomendado. Entonces debemos utilizar otros medios como campus virtual, página web, MOOCs, OCW, enlaces a vídeos, etc. Además, es recomendable, enlazar la bibliografía recomendada desde cada guía docente al servicio de Documentación de la ULL con la finalidad de facilitar al alumnado su búsqueda y consulta. Aunar los contenidos de la asignatura con la bibliografía/recursos a emplear en este mismo apartado de la guía docente, supone mostrar la relación que debe existir entre ambos al estudiantado. 


\section{BLOQUE III: El PROCESO DE APRENDIZAJE EN LA ASIGNATURA}

- Este bloque tiene como finalidad principal guiar al estudiante en su proceso de aprendizaje de la asignatura. Para ello, es necesario establecer claramente las actividades formativas a desarrollar, la metodología que se va seguir y el sistema de evaluación que se va a fijar.

- Se debe señalar que el profesorado no siempre se va a encontrar con el mismo patrón de comportamiento de alumnado ni con el mismo contexto en la asignatura. En este sentido, se propone que este bloque de aprendizaje sea lo suficientemente amplio y flexible como para que el profesorado pueda adaptarlo a la práctica real de su asignatura en función de las distintas variables que puede presentarse en el contexto de la misma (número de alumnado, tipo de alumnado, etc.). Con ello se permitiría la garantía real del aprendizaje de una asignatura, convirtiéndose en una mejor evidencia para acreditar la calidad de la docencia del profesorado y, a nivel general, de la titulación. Esto no significa que no haya que realizar desde el primer momento de la elaboración de la GEA una planificación detallada de las actividades formativas a desarrollar en la asignatura, de la metodología docente y del sistema de evaluación.

- Esto conlleva a plantear el aprendizaje del estudiante como el elemento central del diseño de la enseñanza en el que se requiere de un verdadero trabajo en equipo por parte del profesorado de la asignatura y del área de conocimiento.

\section{Actividades formativas y metodología docente.}

- En este apartado se debe establecer claramente las distintas actividades formativas que se van a seguir en la asignatura, así como el número de créditos que se dedicarán a las mismas y el número de créditos que el estudiante deberá dedicar como trabajo autónomo, con el objetivo de garantizar que el estudiante sea capaz de superar la asignatura con éxito.

- Hay que tener en cuenta que, en la mayoría de los casos, la elección de las actividades formativas y de la metodología docente viene condicionada por la memoria de verificación/modificación de la titulación. Esto no implica que no se pueda realizar una valoración sobre la adecuación de las mismas al contexto real de la asignatura, de manera que puedan establecerse modificaciones futuras en dicha memoria de verificación/modificación de la titulación.

- La metodología docente que orientan las actividades formativas debe ir dirigida a la adquisición de las competencias de la asignatura por parte del estudiante y debe estar muy relacionada con los métodos 
de evaluación que se vayan a emplear para que se pueda medir el grado de consecución de dichas competencias.

- 1 Clases magistrales: se trata de una actividad presencial en la que el docente hará una exposición de los principales contenidos y conceptos relacionados con las competencias a adquirir en esta asignatura. Durante el desarrollo de estas sesiones, el estudiante toma apuntes, plantea dudas, hace sugerencias, etc.

- 2 Trabajo en grupo: a lo largo de la materia hay planificadas una serie de actividades que deberán ser resueltas en grupo. Estas actividades, no solo están pensadas para trabajar determinadas competencias específicas de la materia, sino también para otras competencias genéricas, como la de trabajo en grupo y la toma de decisiones. Aproximadamente, el estudiante tendrá que invertir 2 créditos en esta actividad formativa.

${ }^{-3}$ Trabajo autónomo: durante el desarrollo de la asignatura, los estudiantes tendrán que hacer trabajo individual, como lectura de bibliográfica básica y complementaria, preparación de las clases teóricas y repaso académico para superar las pruebas evaluativas previstas en la asignatura.

- 4 Presentaciones orales: en esta asignatura, las tareas que surjan del trabajo en grupo desarrollado, serán presentadas de manera oral al resto de compañeros. La preparación de estas presentaciones, como la exposición presencial en el aula.

- Tipo de actividades formativas: clases magistrales, resolución de casos prácticos, trabajos en grupo, presentaciones orales, trabajo autónomo (tareas individuales), seminarios, lecturas, debates/discusiones, role playing, estudios de casos, prácticas de laboratorio, etc.

\section{Sistema de EVAlUACIÓN: CRITERIOS Y PORCENTAJES DE CALIFICACIÓN.}

- La evaluación es una herramienta importante del proceso de enseñanza-aprendizaje que más suele interesar al estudiante pues le permite conocer el grado de desarrollo de su aprendizaje. Es por ello que en este apartado debe proporcionarse una información detallada sobre cómo se va a evaluar y qué instrumentos y técnicas de evaluación se van a utilizar, es decir, en definitiva, qué seguimiento y control del aprendizaje se va a seguir.

- En este sentido, el sistema de evaluación permite, al profesorado, comprobar de forma objetiva si el alumnado ha adquirido las competencias establecidas en la GEA y, al alumnado, tener conocimiento de los criterios de evaluación para poder preparar sus asignaturas de forma adecuada. La evaluación puede ser continua o final. En el sistema de evaluación continua se ha de explicar cómo puede superar la asignatura el estudiante, a través de qué tipo de pruebas y la ponderación o peso de cada una de ellas dentro de la nota final de la asignatura. Además, el profesorado debe fijar los criterios que permitan al estudiante aprobar la asignatura y/o conjugar las distintas actividades con la prueba final, 
si la hubiere. También, debe establecer la obligatoriedad, si fuera el caso, de una calificación mínima y/o determinar los requisitos específicos en algunas de las actividades o en todas. La evaluación final se entiende realizada en un solo momento o acto académico y podrá incluir todas aquellas pruebas que el profesorado estime necesarias para adquirir las competencias establecidas en la GEA.

- Se evalúan tanto los conocimientos asimilados como la realización de actividades de diversa índole, por tanto, el tipo de evaluación se establece según el tipo de asignatura y puede consistir en: cuestionarios tipo test, pruebas objetivas sobre el contenido teórico (orales y/o escritas), resolución de problemas, actividades que requieran trabajo empírico, asistencia a clase y participación, pruebas de ensayo, estudio de casos, comentarios de texto, prácticas de laboratorio, otro tipo de prácticas que estime el profesorado, etc.

- En definitiva, tener claro cuáles son las competencias y resultados de aprendizaje establecidos en la asignatura, los contenidos (temario) desarrollados, las actividades formativas y el grado de superación o dominio que debe alcanzar el estudiante, es fundamental. Este hecho vincularía el bloque II de enseñanza con el bloque III de aprendizaje de la GEA, cerrándose el proceso de enseñanza-aprendizaje de la asignatura. Esta interrelación entre los distintos bloques se aprecia en la siguiente figura:

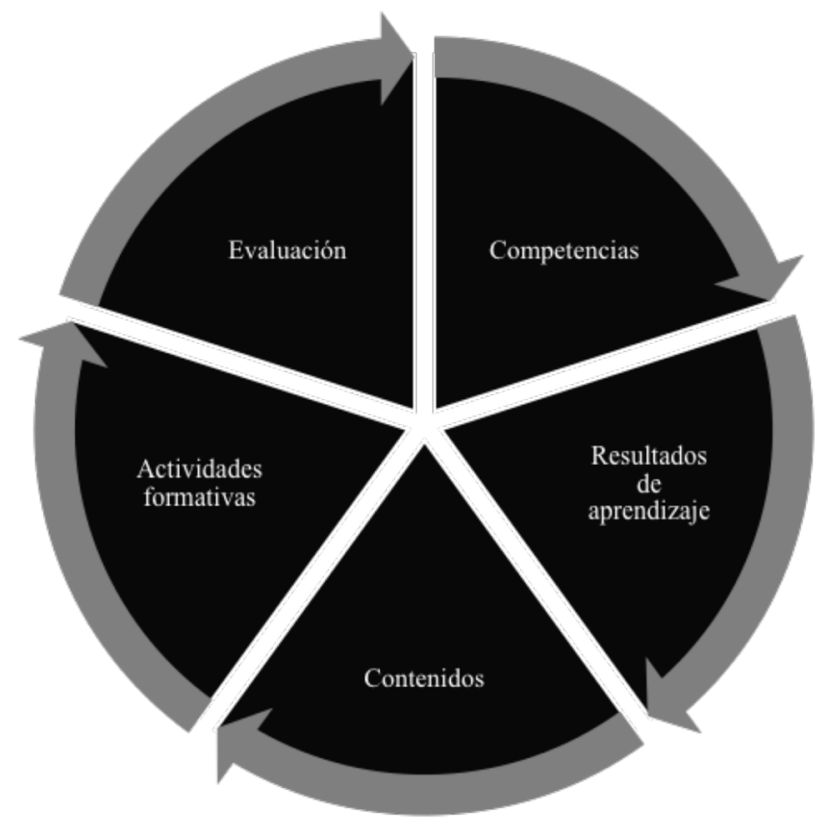

Figura 2. 


\section{EXPERIMENTACIÓN DEL MODELO GEA}

Durante el curso 2017/2018 se diseñaron y se pusieron en práctica distintas actividades formativas para integrar la GEA en el proceso de enseñanza y aprendizaje. Esta experiencia fue llevada a cabo en las asignaturas de Política Regional (Grado de Economía), Orientación y Función Tutorial del Profesorado (Grado de Maestro de Educación Infantil) y Sociedad, Familia y Escuela (Grado de Maestro de Educación Infantil). Con la finalidad de valorar este modelo de guía docente, se llevó a cabo un proceso de evaluación para determinar los beneficios de GEA en los procesos de enseñanza y aprendizaje del alumnado universitario.

- Participantes

- Las personas que participaron en la evaluación de la experimentación del modelo GEA, fueron los estudiantes que, durante el curso $2017 / 2018$, estaban matriculados en las asignaturas donde se llevó a cabo esta experiencia de innovación. De manera más específica, y tal y como se presenta en la siguiente tabla, se contó con una muestra total de 126 estudiantes, con una edad media de 21,34 años $(s d=3,5 ; \min =19 ; \max =39)$, de los cuales el $82,54 \%$ eran mujeres $(n=104)$ y el $17,46 \%$ eran hombres $(n=11)$.

\begin{tabular}{|l|l|}
\hline \multicolumn{2}{|l|}{ Tabla 1.} \\
\hline $\mathrm{n}=126$ & $\underline{x}=21,34 ; \mathrm{Sd}=35 ;$ Min=19; Max=39 \\
\hline Edad & $17,34 \% ; \mathrm{n}=22$ \\
\hline Política regional & $14,29 \% ; \mathrm{n}=18$ \\
\hline $\begin{array}{l}\text { Orientación y función tutorial del } \\
\text { profesorado }\end{array}$ & $68,25 \% ; \mathrm{n}=86$ \\
\hline Sociedad, familia y escuela & \\
\hline
\end{tabular}

\section{INSTRUMENTO DE RECOGIDA DE DATOS}

- Para conocer la opinión que tenían los estudiantes universitarios que participaron en la experiencia, se administró un cuestionario online de elaboración propia adaptado a los objetivos de la evaluación y a los participantes a los que iba dirigido. Tras valorar diferentes alternativas para el envío online de la prueba, se decidió finalmente emplear la aplicación «Formularios» de Google, dado que incluía los requisitos técnicos necesarios para su administración: posibilidad de participación de usuarios pertenecientes exclusivamente a la Universidad de La Laguna, recepción de una única respuesta por parte de cada estudiante, participación masiva de los usuarios en 
el cuestionario, exportación de los resultados a ficheros de hojas de cálculo tipo Excel para su posterior análisis, etc.

- Este instrumento de recogida de información se organizó a partir de preguntas dicotómicas y abiertas. En concreto, el cuestionario pretendía valorar cuestiones como: nivel de desarrollo de los objetivos y competencias propuestas en las materias, aportaciones de la asignatura al perfil profesional del alumnado, estructura y organización del modelo GEA y ventajas e inconvenientes de la GEA.

\section{Procedimiento de Recogida de datos}

- La aplicación del cuestionario de evaluación para valorar la experiencia desarrollada, se llevó a cabo en las últimas sesiones de clase. De manera específica, el profesorado responsable de las asignaturas siguió el siguiente procedimiento:

- En las últimas semanas de clase el profesorado informó a sus estudiantes que recibirían un correo electrónico solicitando la cumplimentación de un cuestionario online para valorar la experiencia desarrollada.

- Una vez informado al alumnado, los docentes enviaron un correo electrónico al alumnado (a través del correo institucional; cuentas aluXXX@ull.edu.es) solicitando su participación voluntaria para cumplimentar el cuestionario diseñado. Por esta misma vía, se les indicó la fecha límite para completar el instrumento de recogida

- Finalizado el plazo de recogida de información, se generó automáticamente una hoja de cálculo para integrar los resultados de las tres asignaturas que participaron en la experiencia.

\section{ANÁLISIS E INTERPRETACIÓN DE LA INFORMACIÓN}

- La información recogida a través del cuestionario fue tratada a través de un análisis de contenido de las preguntas abiertas. Además, este análisis fue completado con un estudio del número de ocurrencias que tenían las respuestas ofrecidas por los participantes en el estudio. Para identificar cada una de las aportaciones de los participantes, se codificó cada relato apartado con la referencia Pn (donde «P» hacía referencia a la palabra participante y «n» representaba el número de encuestado). 


\section{RESULTADOS}

- Los resultados de la evaluación han reflejado que, desde la perspectiva de los estudiantes, la propuesta metodológica empleada permitió alcanzar los objetivos y competencias previstas en la asignatura. En este sentido, una amplia mayoría de estudiantes $(73,0 \%)$ valoró que se han «alcanzado los objetivos y las competencias de manera excepcional» (P2). Para el alumnado restante $(29,9 \%)$, no se cumplieron todas las propuestas formativas establecidas «debido a que muchos de los temas no se han tratado con la profundidad necesaria» (P16) principalmente por «la falta de tiempo» (P21).

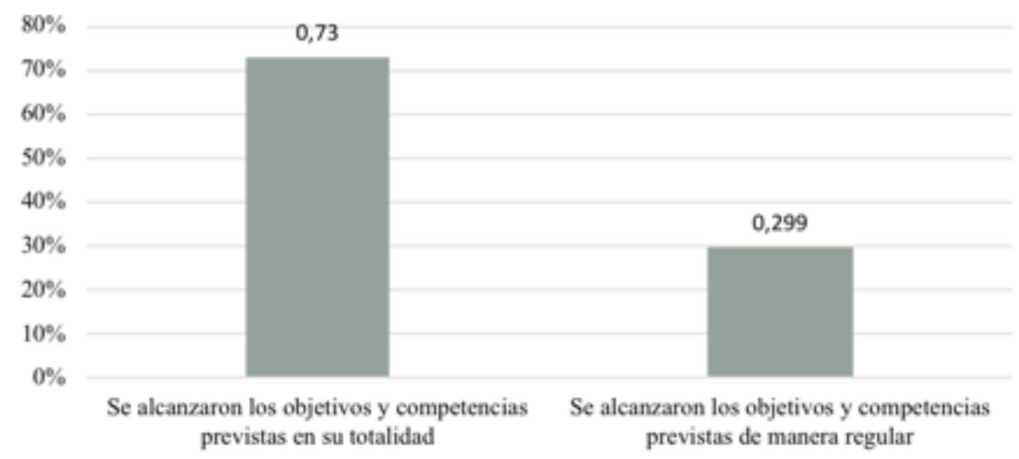

Figura 3.

Por otra parte, los estudiantes destacaron que la estrategia metodológica empleada basada en el nuevo modelo de guía docente elaborada, les permitió conectar los contenidos que estaban trabajando en la asignatura con su futuro desempeño profesional $(93,8 \%)$. Los estudiantes señalaron que «me ha aportado conocimientos y herramientas para mi futura labor como docente» (P30) y que «me ha aportado mucha información que desconocía y la cual estoy segura de que me será de gran utilidad para mi trabajo como docente. Sobre todo, la importancia de la relación de la familia y la escuela y el uso necesario de la tutoría y orientación en los centros» (P57).

- En cuanto a las valoraciones específicas acerca de la estructura de la GEA, el 95,3\% de los estudiantes participantes en la experiencia, consideró que recogía los apartados, la información y el contenido necesario para orientar el proceso de la asignatura. El alumnado manifestó que «es bastante completa» (P15), y está «organizada adecuadamente y de manera más amena al anterior modelo» (P31). De hecho, uno de los estudiantes sugirió que «este formato para la 
Guía Docente es mucho más cómodo, pues te indica bien qué hay en cada apartado (...), además, su estructura (poder abrir y cerrar pestañas), considero que es mucho más cómodo a la hora de buscar información» (P48). El 4,6\% de los encuestados, introdujo algunas sugerencias para mejorar el modelo de guía docente, como es un "apartado dentro de la bibliografía, que permita saber qué referencias son básicas para cada uno de los temas y prácticas a trabajar en la asignatura» (P39) o bien, indicar con mayor precisión «la importancia de realizar la asignatura, ya que la mayoría del alumnado se matricula en asignaturas que les resulten fácil aprobar o que no les ocupe mucho tiempo ni esfuerzo para aprobar y poco se detienen en ver la importancia de cada asignatura y de los conocimientos que te puede aportar para nuestra futuro profesión» (P36).

- En opinión del alumnado, el modelo de guía docente experimentado, aporta una serie de beneficios a su proceso formativo, sobre todo a «a la hora de buscar la información está mejor estructurada y señalada» (P5) y también consideran que «puede aportarme información útil de la asignatura, como por ejemplo los criterios empleados y los contenidos a tratar» (P43). Con relación al aprendizaje «considero que el nuevo modelo de Guía docente facilita al alumnado su trabajo a la hora de organizarse el trabajo autónomo de la signatura» (P2).

- Finalmente, los estudiantes destacaron como principal ventaja del modelo GEA que ofrece orientaciones para enfrentar el proceso de enseñanza y aprendizaje de la asignatura de una manera clara $(62,8 \%)$ y propone información de interés para el desarrollo de la materia $(37,1 \%)$. Así, el alumnado consideró que «con esta guía docente se conoce la metodología, así como los contenidos que se impartirán de la asignatura de manera general y esquematizada» (P21) y «es clara y amena (...), se exponen todos los contenidos de manera que sea entendible fácilmente» (P27).

\section{CONCLUSIONES}

Cada año son muchas las evidencias que ponen de manifiesto la necesidad de mejorar el modelo y estructura de las guías docentes que orientan la enseñanza universitaria. Las guías docentes tendrían que tener en cuenta los requisitos de la normativa vigente, garantizando en todo caso los derechos del alumnado y la calidad de la enseñanza que se imparta. No se puede olvidar que, a la hora de hacer reclamaciones, la guía docente es un documento de referencia muy importante para los estudiantes. Por eso los docentes deberían respetar que el desarrollo de las materias se corresponda con la planificación que se especifica en la guía docente. 
En los trabajos que hemos realizado con estudiantes de la educación superior (Álvarez, González, López, Peláez y Peña, 2017; Álvarez, López, Vázquez y González, 2018), se ha puesto de manifiesto que algunos alumnos y alumnas, cursan asignaturas sin haber tenido contacto con la guía docente. En otros casos, se han recogido opiniones poco favorables hacia la guía docente, dado el carácter administrativo que adquiere en ocasiones la misma y lo poco cercano al proceso formativo de los estudiantes.

La guía docente, como herramienta de la educación superior, ha perdido en muchos casos su sentido y utilidad como referente de la práctica educativa, puesto que en muchos casos no se corresponde lo que figura en la guía docente con el desarrollo del proceso educativo. La propia estructura de las guías docentes tampoco favorece mucho el uso como recurso de apoyo al alumnado. Efectivamente, la guía docente constituye un documento frío y poco cercano a la vida del estudiante, excesivamente recargado de elementos técnicos y con apartados poco atractivos, como las competencias y las estrategias evaluativas. Por otro lado, algunos apartados como el cronograma no tienen mucha utilidad por cuanto supone una previsión con un año de antelación que en la mayoría de los casos no se puede ajustar al verdadero desarrollo de la asignatura. En este sentido falta flexibilidad en el modelo de guía docente, así como la incorporación de otros apartados que sintonicen mejor con las necesidades del alumnado.

En general, las opiniones del alumnado que ha empleado el modelo de guía docente GEA, han sido favorables: han valorado positivamente la guía docente y la consideran un recurso útil, puesto que les permitió conectar los contenidos que estaban trabajando en la asignatura con su futuro desempeño profesional. Asimismo, destacaron positivamente la estructura, puesto que contemplaba los apartados necesarios, la información y el contenido necesario para orientar el proceso de aprendizaje de la misma. Los estudiantes destacaron como principal ventaja del modelo GEA que ofrece orientaciones para enfrentar el proceso de enseñanza y aprendizaje de la asignatura de una manera clara, exponiendo todos los contenidos de manera entendible. La incorporación de apartados como lo que aporta la asignatura al perfil profesional de la titulación fue un aspecto destacado por el alumnado.

\section{REFERENCIAS BIBLIOGRÁFICAS}

Álvarez, P., González, O., LóPez, D., Peláez, P., y Peña, R. (2017). La guía docente como recurso de la enseñanza: análisis de funciones y alternativas innovadoras en la Universidad de La Laguna. VIII Jornadas de Innovación Educativa, Universidad de La Laguna, Tenerife.

Álvarez, P., LóPEZ, D., Vázquez, R., y González, O. (2018). La guía docente en la enseñanza universitaria: valoraciones desde la perspectiva del alumnado. IX Seminario de la Red Interuniversitario de Profesorado de Orientación. Universidad de Valladolid, Valladolid. 
García, M. (2008). Guías docentes de asignaturas de Grado en el EEES. Editum: Universidad de Murcia.

Marcelo, C., Yot, C., Mayor, C., Sánchez, M., Murillo, P., Rodríguez, J., M. y Pardo, A. (2014). Las actividades de aprendizaje en la enseñanza universitaria: żhacia un aprendizaje autónomo de los alumnos? Revista de Educación, 363, 334-359.

Sánchez, M., Ruiz, C., y Pascual, I. (2011). La guía docente como eje del proceso de enseñanza-aprendizaje. Bordón, 63 (2), 53-64.

YANIZ, C. (2006). Planificar la enseñanza universitaria para el desarrollo de competencias. Educatio Siglo XXI, 24, 17 - 34.

Zabalza, M. A. (2003). Competencias docentes del profesorado universitario. Calidad y desarrollo profesional. Madrid: Narcea. 